\title{
Single Fiber Model of Particle Retention in an Acoustically Driven Porous Mesh
}

\author{
Michael T. Grossner \\ Case Western Reserve University \\ Alan E. Penrod \\ Case Western Reserve University \\ Joanne M. Belovich \\ Cleveland State University \\ Donald L. Feke \\ Case Western Reserve University
}

Follow this and additional works at: https://engagedscholarship.csuohio.edu/encbe_facpub

Part of the Chemical Engineering Commons

How does access to this work benefit you? Let us know!

\section{Repository Citation}

Grossner, Michael T.; Penrod, Alan E.; Belovich, Joanne M.; and Feke, Donald L., "Single Fiber Model of Particle Retention in an Acoustically Driven Porous Mesh" (2003). Chemical \& Biomedical Engineering Faculty Publications. 151.

https://engagedscholarship.csuohio.edu/encbe_facpub/151

This Article is brought to you for free and open access by the Chemical \& Biomedical Engineering Department at EngagedScholarship@CSU. It has been accepted for inclusion in Chemical \& Biomedical Engineering Faculty Publications by an authorized administrator of EngagedScholarship@CSU. For more information, please contact library.es@csuohio.edu. 


\title{
Single fiber model of particle retention in an acoustically driven porous mesh
}

\author{
Michael T. Grossner , Alan E. Penrod , Joanne M. Belovich , Donald L. Feke
}

\section{Introduction}

Separation and recovery of the dispersed phase of a particulate suspension is a common requirement in fluids processing. Some techniques that accomplish this task use external fields to improve the separation performance. A new separation method that relies on an acoustic field resonating within a porous mesh having a pore size much larger than the particle diameter has been developed and tested in our laboratory [1,2]. Suspensions flow through the acoustic chamber without a significant pressure drop (because of the large pore size of the mesh) and particles are trapped within the mesh when the acoustic field is activated. The small pressure drop results in decreased pumping costs compared to conventional filtration processes.

Overall particle retention efficiencies as high as $90 \%$ have been reported [1]. In addition, laboratory experiments have revealed a number of interesting phenomena associated with the motion of particles through the filter mesh. These include the apparent focusing of particles along specific trajectories within the mesh, and the stable levitation of clusters of particles at positions that do not contact any part of the solid mesh despite continued fluid flow.

In order to more thoroughly understand these observed phenomena and the particle retention mechanism, and also to be able to predict the performance of the experimental apparatus, a mathematical model was created based on acoustic and transport theory. The model presented in this paper examines the acoustic and flow forces near a single fiber of the mesh. Using the MATLAB programming language, a graphical interface was developed that allows the user to input operating parameters and see the results of the calculations, i.e., particle trajectories and flow fields near the filter element.

The following section of this paper briefly describes the experimental apparatus along with a typical procedure for its operation. Section 3 illustrates the theory of the model (Appendix A adds mathematical detail to this description), while Sections 4 and 5 give some 
visualizations and explanations of results. Finally, a summary is presented in Section 6 including the direction of future endeavors.

\section{Experimental}

A schematic of the experimental chamber is given in Fig. 1. The chamber is composed of a Plexiglas structure supporting a piezoelectric transducer and a glass reflector. A resonating ultrasonic plane wave (at a frequency typically near $1 \mathrm{MHz}$ ) is established in the enclosed volume, which also houses the porous mesh. A typical mesh is composed of polyester and contains about 30 pores per inch, but meshes of other materials and pore sizes have been studied.

Fluid containing small particles (tens of microns in diameter) is pumped through the chamber at a slow $(\sim 1$ $\mathrm{mm} / \mathrm{s}$ ) rate. Particles are retained when the acoustic field is active, but pass through the chamber when the transducer is not powered. This allows for easy cleaning and regeneration of the media or recovery of the retained solids, as the chamber can be flushed when no acoustic field is present.

Fig. 2 contains photographs showing a suspension of polystyrene particles flowing through the porous mesh (black) with no acoustic field (a) and with a resonant ultrasonic field (b). Note the significant retention of the white particles in the latter case. Experimental studies in which the effects of mesh pore size, acoustic energy, and flow configuration on the particle capture efficiency have been reported [l 13$]$.

It is possible to postulate a few modes by which the combination of acoustic and hydrodynamic effects can cause the particles to be retained within the mesh. In one, the particle may simply collide and stick with an element of the mesh. It has been observed experimentally that, in some regions, particles can be focused along specific trajectories and that this results in the formation of dendritic structures attached to fixed

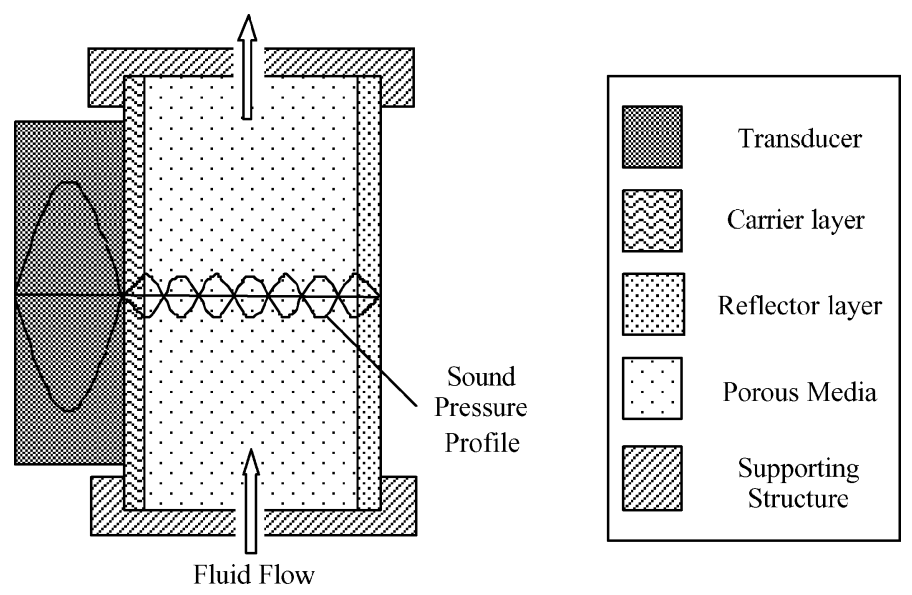

Fig. 1. A schematic of the experimental chamber.
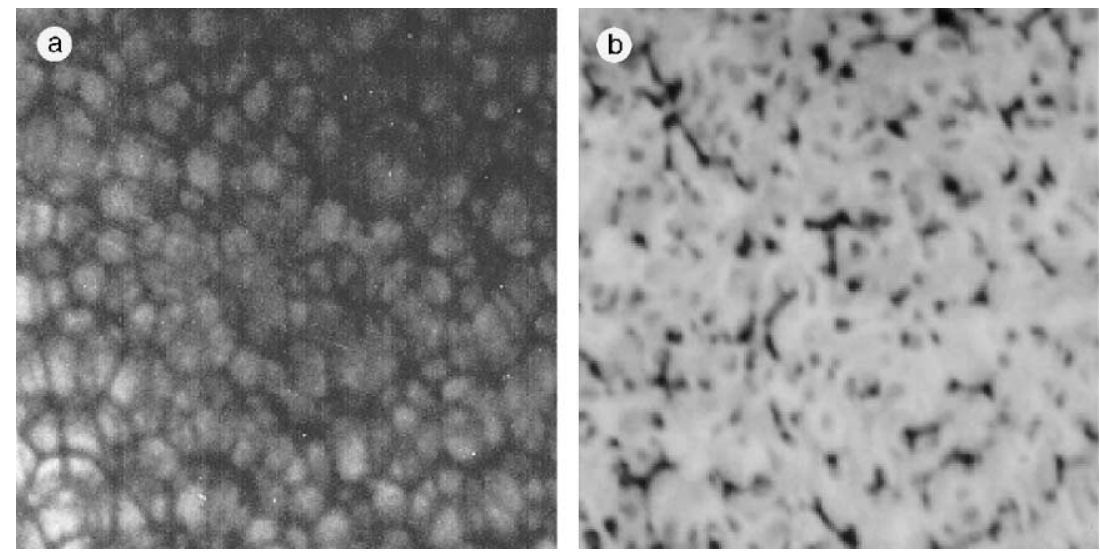

Fig. 2. A photograph of the porous mesh (solid black elements) with a suspension of particles flowing through. No sound energy is present in (a); the ultrasonic standing wave is active in (b). Note the particle accumulation (the white regions in (b)) in the presence of the acoustic field. 
points on the mesh. Another possible collection mode is that the interaction of the acoustic field with the porous media may create points where particles are held in the fluid solely by acoustic forces, a phenomenon that has been observed in the experiments. A third collection method would be for particles to be trapped by agglomerating with other particles, forming temporary flocs too big to pass through the necks between pores.

To understand the theoretical basis for these various mechanisms and experimental observations, we have constructed a mathematical model that attempts to predict the motion of particles in response to the acoustic and flow forces present within the mesh. If proven successful, such a model can form the foundation for optimization of the device design and selection of process parameters.

\section{Model description}

Since the geometry of the mesh is complex, we adopt the standard modeling practice of studying the region surrounding one element of the porous mesh. Here, we picture the mesh to be an assembly of cylinders. A qualitative description of the model equations that describes the particle trajectories is given in this section. For a full derivation and additional mathematical detail, see Appendix A.

The model intends to provide the two-dimensional trajectories of particles in the vicinity of one mesh element, assumed to be an infinite circular cylinder. The axis of the cylinder fiber is perpendicular both to the upstream flow direction and to the direction of the incident ultrasonic plane wave. The angle between the directions of the approach flow and the acoustic field, however, is arbitrary. Fig. 3 defines the coordinate systems used for the trajectory calculations. The fluid flow is described using $(x, y)$ coordinates, with the approach flow always in the $+x$ direction. We also use the polar angle $\phi$ measured from the $+x$ direction for use in de-

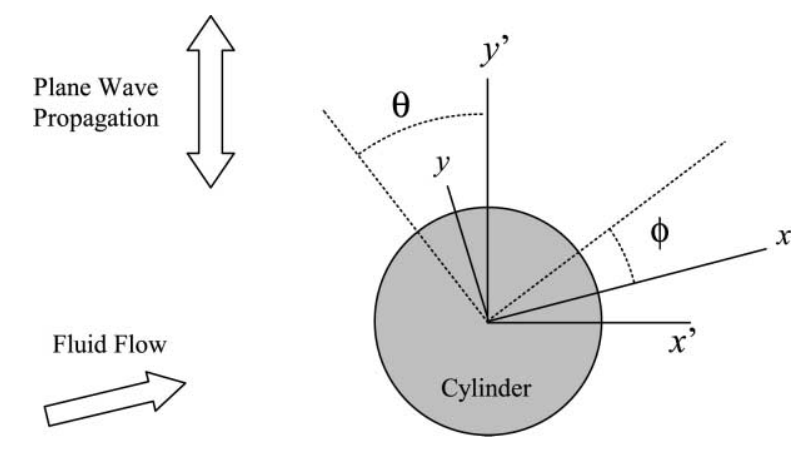

Fig. 3. Coordinate system and orientation of cylinder, incident acoustic wave, and fluid flow for trajectory simulations. scribing the hydrodynamic flow field. The acoustic field is described using $\left(x^{\prime}, y^{\prime}\right)$ coordinates, with the incident acoustic field propagating in the $y^{\prime}$-direction. The polar angle $\theta$ is measured from the $+y^{\prime}$-direction.

At each point in the fluid, a balance between acoustic and drag forces determines the direction and magnitude of the particle velocity. Since the particles are small, the net force on the particles is assumed to be zero. Other major assumptions include creeping flow around the cylinder, no interparticle hydrodynamic or acoustic interactions, and steady state particle velocities.

The following equations describe the particle motion for the case of orthogonal approach flow and incident acoustic field (zero offset between the $x$ and $x^{\prime}$ axes):

$$
\begin{aligned}
\frac{\mathrm{d} x_{\mathrm{p}}^{*}}{\mathrm{~d} t^{*}}= & \frac{2 G_{k}}{3}\left[\frac{\partial U^{*}}{\mathrm{~d} v} \sin \theta+\frac{1}{v} \frac{\partial U^{*}}{\mathrm{~d} \theta} \cos \theta\right] \\
& +\frac{\ln r^{*}-0.5\left(1-1 / r^{* 2}\right) \cos 2 \phi}{2.002-\ln R e_{\mathrm{c}}}
\end{aligned}
$$

and

$$
\begin{aligned}
\frac{\mathrm{d} y_{\mathrm{p}}^{*}}{\mathrm{~d} t^{*}}= & -\frac{2 G_{k}}{3}\left[\frac{\partial U^{*}}{\mathrm{~d} v} \cos \theta-\frac{1}{v} \frac{\partial U^{*}}{\mathrm{~d} \theta} \sin \theta\right] \\
& -0.5 \frac{\left(1-1 / r^{* 2}\right) \sin 2 \phi}{2.002-\ln R e_{\mathrm{c}}} .
\end{aligned}
$$

Here, dimensionless variables (indicated by $*$ )

$x_{\mathrm{p}}^{*}=\frac{x_{\mathrm{p}}}{R_{\mathrm{c}}}, \quad y_{\mathrm{p}}^{*}=\frac{y_{\mathrm{p}}}{R_{\mathrm{c}}}, \quad r^{*}=\frac{r}{R_{\mathrm{c}}}, \quad$ and $\quad t^{*}=\frac{U_{\mathrm{f}} t}{R_{\mathrm{c}}}$

are used. The variables $x_{\mathrm{p}}$ and $y_{\mathrm{p}}$ represent the coordinates of the particle while $r$ and $v(=k r$, where $k$ is the wave number of the acoustic field) also denote the distance from the center of the cylinder. The Gor'kov number,

$G_{k}=\frac{R_{\mathrm{p}}^{2} k E_{\mathrm{ac}}}{\mu U_{\mathrm{f}}}$,

represents the ratio of acoustic forces to hydrodynamic drag forces on the particles while the Reynolds number based on the cylinder diameter

$R e_{\mathrm{c}}=\frac{2 U_{\mathrm{f}} R_{\mathrm{c}} \rho_{\mathrm{f}}}{\mu}$

characterizes the flow strength. The radius of the particle and the cylinder (filter element) are $R_{\mathrm{p}}$ and $R_{\mathrm{c}}$, respectively. The fluid velocity far upstream is $U_{\mathrm{f}}$; the fluid has viscosity $\mu$ and density $\rho_{\mathrm{f}}$. Standard expressions for the creeping motion around a cylinder are used to describe the flow field. Since these are approximations, the resulting equations that describe the motion of the particles (Eqs. (1) and (2)) are strictly valid only near the cylinder. The acoustic energy density is $E_{\mathrm{ac}}$ and $U^{*}$ is the dimensionless acoustic potential, which depends on the position of the cylinder relative to the incident 
acoustic field. See Appendix A for a more detailed definition of $U^{*}$.

\section{Results: particle capture plots}

Given the above system of equations, particle motion can be computed at each point in space, and these motions can be integrated to predict particle trajectories in the vicinity of the cylinder. A simple substitution method is used to solve the set of differential equations to obtain these trajectories. Stopping conditions include a particle impinging upon the fiber element, leaving the selected area of interest, or levitating at a point in space.

Although the model allows for all variables to be adjusted, the results presented here show only the effect of varied cylinder positions (with respect to the incident acoustic field), angle of the fluid flow, and intensity of the acoustic field. The physical parameters used in the model, chosen to match those in typical experiments, are listed in Table 1. These parameters result in a $R e_{\mathrm{c}}$ of 0.1 .

With no acoustic forces, the particles merely trace the motion of the fluid around the cylinder, as shown in Fig. 4(a). This figure shows both local and integrated particle trajectories. The circle in the center of the plot is the cross-section of the cylinder. The field of arrows in the background is the direction of particle motion if the particle was located at the position corresponding to the tail of the arrow. The solid curves indicate the paths particles will follow if placed at the upstream end (the left edge) of the curve.

When including the effects of the ultrasonic field, particles are shown to collide with the cylinder (Fig. 4(b)) where they are presumed to stick upon contact.

Table 1

Physical parameters

\begin{tabular}{ll}
\hline Acoustic field & \\
Energy density $\left(E_{\mathrm{ac}}\right)$ & $0.5 \mathrm{~J} / \mathrm{m}^{3}$ \\
Frequency $(\omega / 2 \pi)$ & $1 \mathrm{MHz}$ \\
Fluid: pure water & \\
Bulk velocity $\left(U_{\mathrm{f}}\right)$ & $0.5 \mathrm{~mm} / \mathrm{s}$ \\
Viscosity $(\mu)$ & $0.001 \mathrm{~kg} / \mathrm{m} \mathrm{s}$ \\
Density $\left(\rho_{\mathrm{f}}\right)$ & $1000 \mathrm{~kg} / \mathrm{m}^{3}$ \\
Longitudinal sound speed $\left(c_{\mathrm{f}}\right)$ & $1480 \mathrm{~m} / \mathrm{s}$ \\
Filter element: polyester & \\
Radius $\left(R_{\mathrm{c}}\right)$ & $0.1 \mathrm{~mm}$ \\
Density $\left(\rho_{\mathrm{c}}\right)$ & $1230 \mathrm{~kg} / \mathrm{m}^{3}$ \\
Longitudinal sound speed $\left(c_{1}\right)$ & $2430 \mathrm{~m} / \mathrm{s}$ \\
Shear sound speed $\left(c_{2}\right)$ & $1200 \mathrm{~m} / \mathrm{s}$ \\
Particles: polystyrene & \\
Radius $\left(R_{\mathrm{p}}\right)$ & $10 \mu \mathrm{m}$ \\
Density $\left(\rho_{\mathrm{p}}\right)$ & $1050 \mathrm{~kg} / \mathrm{m}^{3}$ \\
\hline
\end{tabular}

This simulation case has a pressure node of the incident standing wave placed directly at the center of the cylinder. Since the particles have properties that result in motion toward the pressure nodes of the acoustic field, this configuration is expected to be advantageous for particle collection.

Fig. 4(c) shows a magnified region of Fig. 4(b). Here the two particle capture methods mentioned in the previous section can be visualized. At a spot just upstream of the cylinder, particles are trapped, levitated in the fluid by a combination of acoustic and drag forces. The other capture scheme is shown as particles are impinged on the surface of the cylinder.

In contrast, one might expect that locating the cylinder on an antinode of the incident acoustic field would be detrimental to particle capture since particles would be driven away from these positions. However, Fig. 4(d) shows that particles will continue to be captured even in this case. This result stems from the interaction between the incident wave and the waves reflected from the cylinder; the combination actually attracts particles to the filter element at some locations. In order to better visualize the effect of the overall acoustic field, Fig 5(a) presents the results when the flow speed is set to zero. Note that the overall acoustic field does direct particles to certain locations on the cylinder, even though the cylinder is positioned at the antinode of the incident field.

When the cylinder is positioned off of both acoustic nodes and antinodes of the incident field, a more complicated trajectory pattern results (see Fig. 5(b)). In this particular case, there are four distinct spots on the cylinder surface to which particles from a range of origination points are attracted. This focusing is frequently seen in the model results and explains the formation of particle dendrites that have been observed in experiments [4].

Fig. 5(d) shows that particles entering with a flow angle of $45^{\circ}$ with respect to the incident acoustic field can still be captured. Particle trajectories in this simulation are focused to the same points on the cylinder as Fig. 5(b), again indicating that the acoustic fields are the driving force for particle retention.

\section{Results: capture window}

In order to compare the effectiveness of the filter element, it is beneficial to devise a metric for evaluating capture performance. Consider a cross-sectional area perpendicular to the bulk flow direction and upstream from the filter element. There exists a "capture window" in this plane through which all particles that eventually collide with the cylinder pass. The width of the capture window can be used as a measure of the capture 

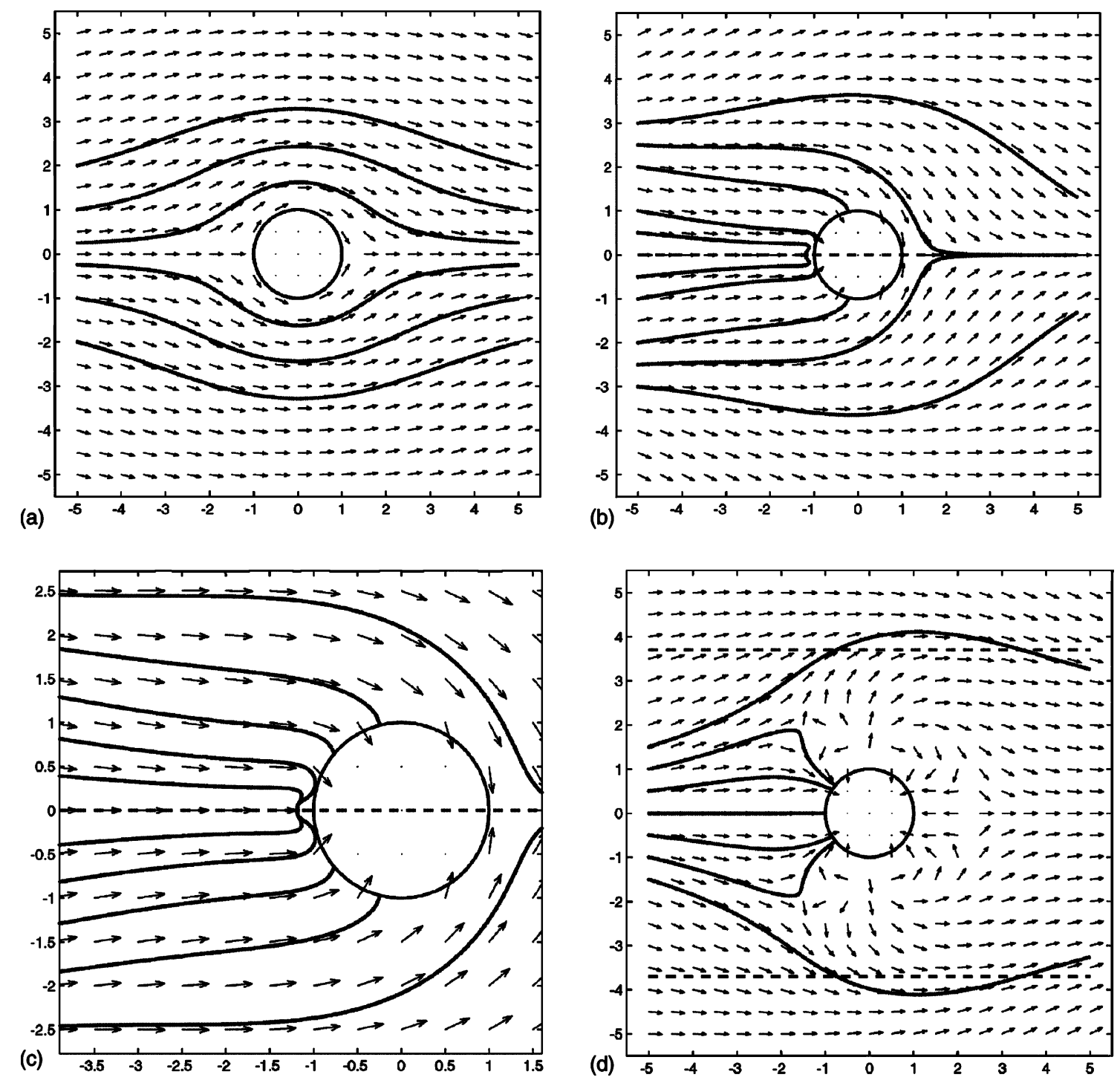

Fig. 4. Model simulation results. The circle is the cross section of a single mesh element, the direction of suspension flow is from left to right, and the ultrasonic wave resonates between the top and bottom with the nodal plane of the incident field intersecting the visual plane at $90^{\circ}$. The unit of length in all results is the radius of the cylinder $(0.1 \mathrm{~mm})$. Arrows indicate the direction of particle motion at a grid point; the solid lines are particle trajectories. The dashed line is the position of the pressure node. No sound field is present in (a). A sound field with a pressure node at the cylinder center is present in (b). The results in (c) show a magnified view of (b); note the position along the node near the fiber where a particle will be suspended in the liquid by a balance of acoustic and drag forces. With the pressure nodes away from the filter element, particle capture may still occur as shown in (d). The value of $G_{k}$ in (b), (c), and (d) is 0.425 .

efficiency under different experimental conditions. For example, the width of the capture window in the case of Fig. 5(b) is about 3 cylinder radii for an acoustic energy density of $0.5 \mathrm{~J} / \mathrm{m}^{3}$ (corresponding to a Gor'kov number of 0.42 for these conditions). If the acoustic energy density is doubled to $1 \mathrm{~J} / \mathrm{m}^{3}$ (Fig. 5(c)), the width of capture increases to more than 5 cylinder radii.

Fig. 6 shows how the width of the capture window varies with $G_{k}$ for three different nodal positions for the case of perpendicular flow and incident acoustic field. The capture widths were calculated by evaluating particle trajectories at each set of data $\left(G_{k}\right.$, nodal position), which corresponds to one point on the plot. The small jumps in the plot are due to imprecision in the width of the capture window, which is calculated to a \pm 0.0625 radii tolerance.

As seen in Fig. 6, the performance of the filter element in each case hits a plateau before $G_{k}$ reaches a value of 2 . Increasing the acoustic energy beyond this point does not significantly increase the width of the capture window. When the pressure antinode is at the cylinder axis, the plateau value decays slightly with $G_{k}$ and will eventually reach zero. This effect is expected, since, as the acoustic energy is increased, particles will be 

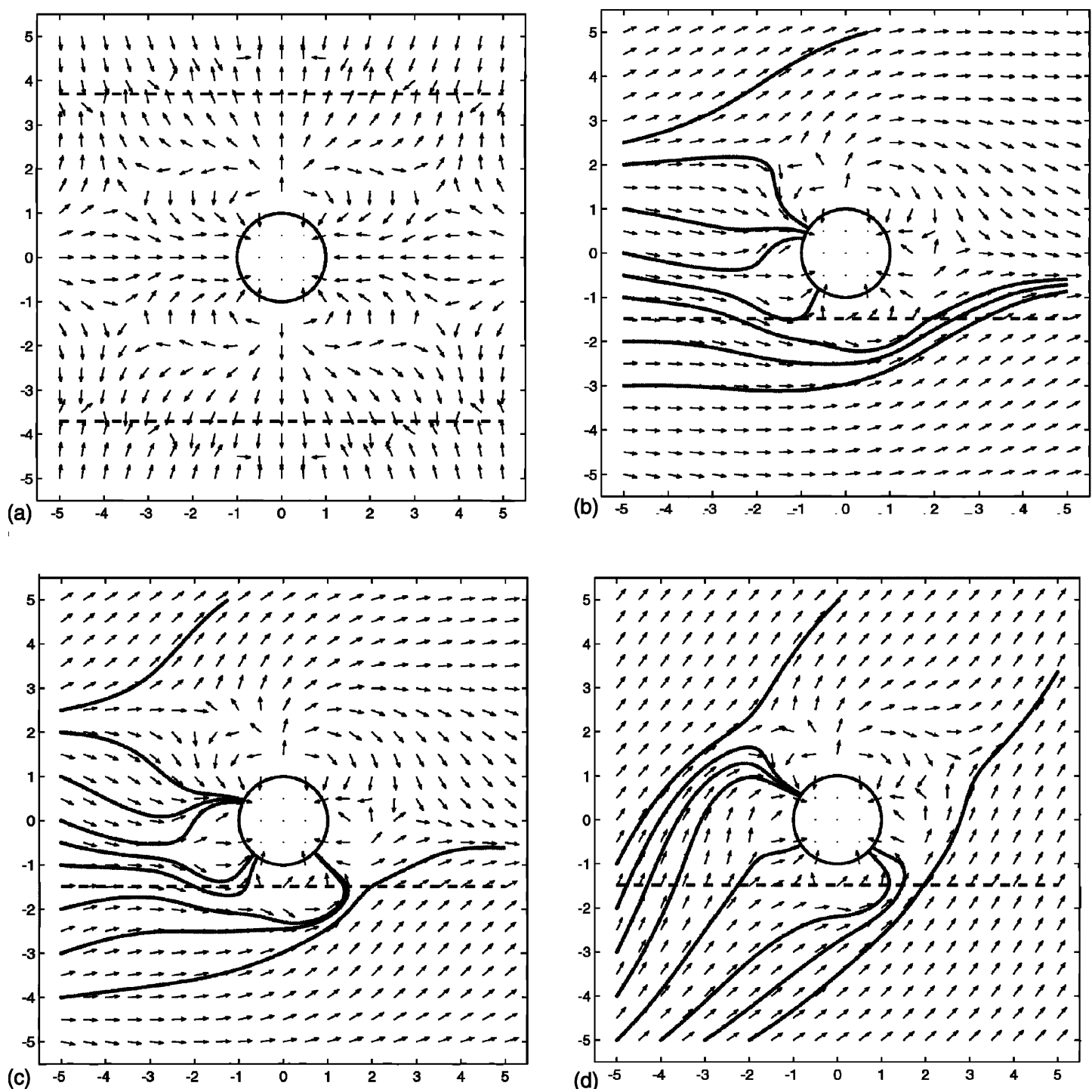

Fig. 5. With no flow, one can visualize only acoustic forces in (a). The simulation resulting in (b) is identical to (c) except that the acoustic energy is doubled in (c), allowing more particles to be collected. (The value of $G_{k}$ is doubled from 0.425 in (b) to 0.850 in (c).) At a flow angle $45^{\circ}$ to the plane wave, particles still tend to flow toward the filter element (d). For (d), $G_{k}$ is 0.425 .

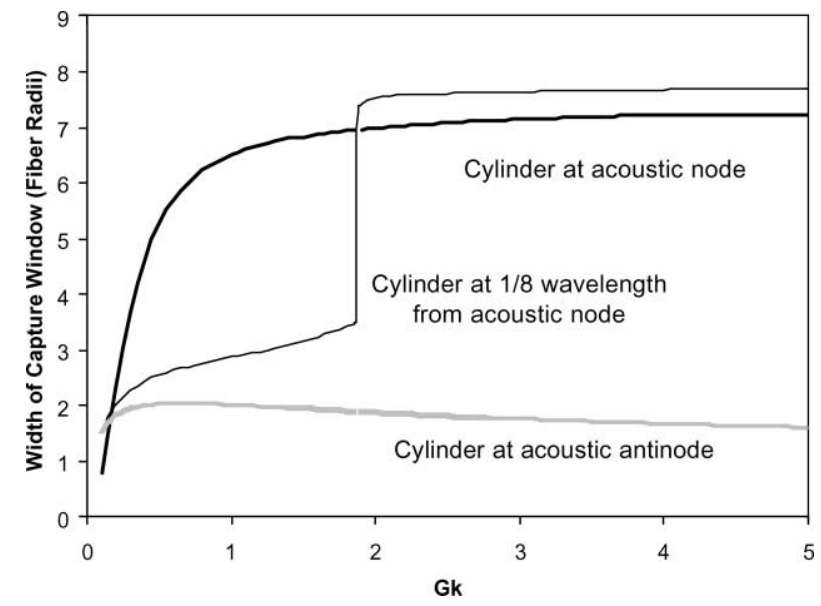

Fig. 6. The width of the capture window as a function of $G_{k}$ for three different positions of the pressure node of the acoustic plane wave. forced to the pressure nodes (and away from the filter element) with greater intensity. For each of the other two cases, the capture width remains constant at large values of $G_{k}$.

One feature seems unusual in Fig. 6, the large increase in capture width near $G_{k}=1.85$ for the case of the node placed 1/8 wavelength from the cylinder axis. It is useful to explain this with the visual aid of trajectory plots. Fig. 7 shows the particle trajectories for $G_{k}=1.85$ and $G_{k}=1.9$; the capture widths are 3.4 and 7.4 fiber radii, respectively. Just below the pressure node, the particle trajectories combine into one streamline. The critical point for capture of this streamline lies between a $G_{k}$ of 1.85 and 1.9 .

All of the particle trajectories used for visualization and for capture window calculations begin at a position located 5 fiber radii upstream from the cylinder axis. 


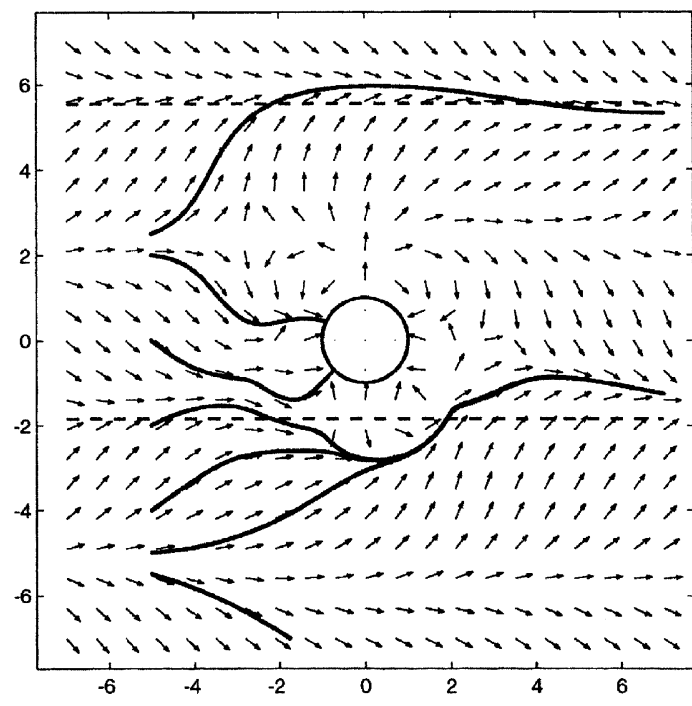

(a)

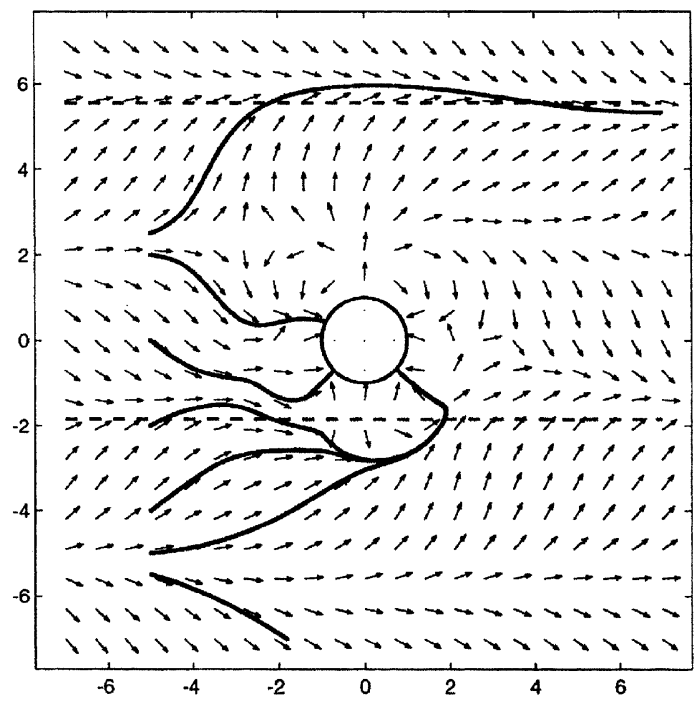

(b)

Fig. 7. With only a small change in $G_{k}$, from 1.85 (a) to 1.9 (b), the width of the capture window more than doubles. The large effect is due to many trajectories combining into one stream of particles that flows by the filter element in the former and is captured in the latter.

The area of the capture window would change if the starting position of the particles were changed to be more upstream or downstream from the filter element because the force due to the incident plane wave is always present. Since the particles are believed to be well dispersed between pores in the mesh, a value of 5 radii (corresponding to $0.5 \mathrm{~mm}$, in this case) was chosen, corresponding to the midpoint between to filter elements in a 25 pore per inch mesh.

\section{Summary}

The single-element modeling approach is useful to see how suspended particles flowing around a cylinder respond to an ultrasonic field. Numerical results indicate that the experimentally observed capture of particles in the mesh is due to the interaction of the incident plane waves with waves reflected from the fiber. This information provides insight into the operation of the acoustic filtration device. These modeling results form part of a comprehensive model of the filtration process, which could ultimately be used to make predictions of overall capture efficiency, as has been done in studies of high gradient magnetic separation processes [5].

\section{Acknowledgement}

This work was supported by NASA through grant NRA-97-HEDS-02-100.

\section{Appendix A}

\section{A.1. General force balance}

Several assumptions are adopted for the derivation of the particle trajectory equations: (1) creeping flow around the cylinder, i.e., $R e_{\mathrm{c}}<1$; (2) the flow field is unaffected by the presence of the particles; (3) gravitational effects are negligible; (4) the particle concentration is dilute so that there are no interparticle hydrodynamic or acoustic interactions; (5) the mass of particles is small enough that inertia is negligible; (6) secondary acoustic forces are ignored; and (7) the fluid flows at steady state. Given these assumptions, the overall force balance on a particle gives

$\boldsymbol{F}_{\mathrm{ac}}+\boldsymbol{F}_{\mathrm{D}}=0$,

where $\boldsymbol{F}_{\text {ac }}$ is the primary acoustic force and $\boldsymbol{F}_{\mathrm{D}}$ is the hydrodynamic drag force.

\section{A.2. Coordinate system for trajectory analysis}

Fig. 3 depicts the coordinate system used in the derivation of the trajectory model equations for the case of orthogonal flow and incident acoustic fields. The flow is defined to always be in the positive $x$ direction, with $\phi$ being the polar angle from the flow direction in cylindrical coordinates. For the description of the acoustic field, we use $\theta$ as the polar angle for the acoustic field, which propagates in the $\theta=0$ direction. 


\section{A.3. Hydrodynamic drag force}

A.3.1. Drag on a sphere in stokes flow

The drag force acting on a sphere under creeping flow conditions is given by

$\boldsymbol{F}_{\mathrm{D}}=6 \pi \mu\left(\boldsymbol{u}_{\mathrm{f}}-\boldsymbol{u}_{\mathrm{p}}\right) R_{\mathrm{p}}$,

where $\mu$ is the fluid viscosity, $\boldsymbol{u}_{\mathrm{f}}$ is the fluid velocity, $\boldsymbol{u}_{\mathrm{p}}$ is the particle velocity, and $R_{\mathrm{p}}$ is the radius of the spherical particle. This solution is accurate for Reynolds numbers less than 0.1 and is accurate to $5 \%$ for Reynolds numbers up to one [6].

\section{A.3.2. Lamb's approximate solution to creep flow about a cylinder}

Lamb provides a solution for creeping flow around an infinite cylinder in Cartesian coordinates [7]. The $x$ and $y$ velocities ( $u_{\mathrm{f} x}$ and $u_{\mathrm{f} y}$, respectively) of the flow are

$u_{\mathrm{f} x}=\frac{1}{2} C\left[\gamma-\frac{1}{2}+\ln \frac{1}{2} \kappa r+\frac{1}{2}\left(r^{2}-R_{\mathrm{c}}^{2}\right) \frac{\partial^{2}}{\partial x^{2}} \ln \frac{r}{R_{\mathrm{c}}}\right]+U_{\mathrm{f}}$,

$u_{\mathrm{f} y}=\frac{1}{4} C\left[\left(r^{2}-R_{\mathrm{c}}^{2}\right) \frac{\partial^{2}}{\partial x \partial y} \ln \frac{r}{R_{\mathrm{c}}}\right]$,

where $\gamma$ is Euler's number $\left(\gamma=0.5772\right.$ [8]), $R_{\mathrm{c}}$ is the cylinder radius, and $r$ is the radial coordinate. The variables $C$ and $\kappa$ are defined by

$C=\frac{2 U_{\mathrm{f}}}{\frac{1}{2}-\gamma-\ln \frac{1}{2} \kappa R_{\mathrm{c}}}$,

$\kappa=\frac{U_{\mathrm{f}} \rho_{\mathrm{f}}}{2 \mu}$.

From the relationship between the Cartesian and cylindrical coordinates, it can readily be shown that

$\frac{\partial^{2}}{\partial x^{2}} \ln r=-\frac{\cos 2 \phi}{r^{2}}$

and

$\frac{\partial^{2}}{\partial x \partial y} \ln r=-\frac{\sin 2 \phi}{r^{2}}$.

Substituting Eqs. (A.4) (A.6) into Eq. (A.3), the flow velocity becomes

$u_{\mathrm{f} x}=U_{\mathrm{f}} \frac{\ln \frac{r}{R_{\mathrm{c}}}-0.5\left(1-R_{\mathrm{c}}^{2} / r^{2}\right) \cos 2 \phi}{2.002-\ln R e_{\mathrm{c}}}$

and

$u_{\mathrm{f} y}=\frac{-0.5 U_{\mathrm{f}}\left(1-R_{\mathrm{c}}^{2} / r^{2}\right) \sin 2 \phi}{2.002-\ln R e_{\mathrm{c}}}$.

\section{A.4. Primary acoustic force}

\section{A.4.1. General equations}

The results of Gor'kov [9], corrected by Barmatz and Collas [10], are used to determine the primary acoustic force. The primary acoustic force is given by the negative gradient of the acoustic potential $U$,

$\boldsymbol{F}_{\mathrm{ac}}=-\nabla U$,

where

$U=2 \pi R_{\mathrm{p}}^{3} \rho_{\mathrm{f}}\left(\frac{\left\langle P^{2}\right\rangle}{3 \rho_{\mathrm{f}}^{2} c_{\mathrm{f}}^{2}} f_{1}-\frac{\left\langle V^{2}\right\rangle}{2} f_{2}\right)$.

Here $c_{\mathrm{f}}$ is the longitudinal sound speed in the fluid, $P$ and $V$ the local pressure and velocity in the fluid at the location of the particle, and \langle\rangle denotes a time average. The factors $f_{1}$ and $f_{2}$ are given by

$f_{1}=1-\frac{c_{\mathrm{f}}^{2} \rho_{\mathrm{f}}}{c_{\mathrm{p}}^{2} \rho_{\mathrm{p}}}$,

$\left.f_{2}=2 \frac{\rho_{\mathrm{p}}-\rho_{\mathrm{f}}}{2 \rho_{\mathrm{p}}+\rho_{\mathrm{f}}}\right)$,

where $\rho_{\mathrm{p}}$ is the particle density and $c_{\mathrm{p}}$ is the longitudinal sound speed in the particle. The factors have allowable ranges of $-\infty<f_{1} \leqslant 1$ and $-2 \leqslant f_{2} \leqslant 1$.

The local pressure and velocity can be written from the real portion of the velocity potential $\Phi$,

$$
\begin{aligned}
& P=\operatorname{Re}\left(-\rho_{\mathrm{f}} \frac{\partial \Phi}{\partial t}\right), \\
& \boldsymbol{V}=\operatorname{Re}(\nabla \Phi) .
\end{aligned}
$$

\section{A.4.2. Acoustic velocity potential around a cylinder}

Hasegawa et al. [11] have developed a velocity potential for a plane progressive wave incident upon a cylinder. The velocity potential of an incident plane standing wave is represented by

$\Phi_{\mathrm{i}}=A \exp (\mathrm{i} \omega t) \sum_{n}^{\infty} \varepsilon_{n}(-\mathrm{i})^{n} \delta_{n} J_{n}(k r) \cos (n \theta)$,

where the term $\delta_{n}$ transforms Hasegawa's expression for progressive waves into a standing wave and

$$
\begin{aligned}
\varepsilon_{n} & =\left\{\begin{array}{ll}
1 & \text { for } n=0 \\
2 & \text { for } n=1,2,3, \ldots
\end{array}\right. \text { and } \\
\delta_{n} & =\mathrm{e}^{-\mathrm{i} k h}+(-1)^{n} \mathrm{e}^{\mathrm{i} k h} .
\end{aligned}
$$

Here $\theta$ is the angle relative to the direction of propagation of the acoustic field, $r$ is the radial coordinate, $A$ is the amplitude of the potential, $h$ is the distance between the nodal plane and the axis of the cylinder, $\omega$ the angular frequency, and $k$ the wavenumber. $J_{n}$ is the cylindrical Bessel function of the first kind and order $n$. Making the simplifications

$B_{n}=\varepsilon_{n}(-\mathrm{i})^{n} \delta_{n} \quad$ and $\quad v=k r$, 
Eq. (A.13) can be rewritten as

$\Phi_{\mathrm{i}}=A \exp (\mathrm{i} \omega t) \sum_{n=0}^{\infty} B_{n} J_{n}(v) \cos (n \theta)$.

The scattered field potential is written as

$\Phi_{\mathrm{s}}=A \exp (\mathrm{i} \omega t) \sum_{n 0}^{\infty} B_{n} d_{n} H_{n}^{(2)}(v) \cos (n \theta)$

with

$H_{n}^{(2)}(v)=J_{n}(v)-\mathrm{i} Y_{n}(v)$,

where $Y_{n}(v)$ is the cylindrical Bessel function of the second kind and order $n$. The coefficients $d_{n}$ are given by Faran [12] and are recast by Hasegawa et al. [11] with algebraic errors. The corrected coefficients are presented here for convenience:

$d_{n}=-\frac{F_{n} J_{n}(x)-x J_{n}^{\prime}(x)}{F_{n} H_{n}^{(2)}(x)-x H_{n}^{\prime(2)}(x)}$,

where

$F_{n}=\frac{\rho_{\mathrm{f}} x_{2}^{2}}{2 \rho_{\mathrm{c}}} \frac{\left(\widehat{A}_{n}-\widehat{B}_{n}\right)}{\left(\widehat{C}_{n}-\widehat{D}_{n}\right)}$

with

$$
\begin{aligned}
\widehat{A}_{n} & =-\frac{x_{1} J_{n}^{\prime}\left(x_{1}\right)}{J_{n}\left(x_{1}\right)-x_{1} J_{n}^{\prime}\left(x_{1}\right)}, \\
\widehat{B}_{n} & =\frac{2 n^{2} J_{n}\left(x_{2}\right)}{n^{2} J_{n}\left(x_{2}\right)-x_{2} J_{n}^{\prime}\left(x_{2}\right)+x_{2}^{2} J_{n}^{\prime \prime}\left(x_{2}\right)}, \\
\widehat{C}_{n} & =-\frac{\left(\frac{x_{2}^{2}}{2}-x_{1}^{2}\right)\left(J_{n}\left(x_{1}\right)-J_{n}^{\prime \prime}\left(x_{1}\right)\right)}{J_{n}\left(x_{1}\right)-x_{1} J_{n}^{\prime}\left(x_{1}\right)}, \\
\widehat{D}_{n} & =\frac{2 n^{2}\left(J_{n}\left(x_{2}\right)-x_{2} J_{n}^{\prime}\left(x_{2}\right)\right)}{n^{2} J_{n}\left(x_{2}\right)-x_{2} J_{n}^{\prime}\left(x_{2}\right)+x_{2}^{2} J_{n}^{\prime \prime}\left(x_{2}\right)} .
\end{aligned}
$$

Here $\rho_{\mathrm{c}}$ is the density of the cylinder and $x, x_{1}$ and $x_{2}$ are given by

$x=k R_{\mathrm{c}}, \quad x_{1}=\frac{\omega R_{\mathrm{c}}}{c_{1}}, \quad$ and $\quad x_{2}=\frac{\omega R_{\mathrm{c}}}{c_{2}}$

with $c_{1}$ and $c_{2}$ being the velocities of longitudinal and shear waves in the cylinder material, respectively. Writing the velocity potential as the sum of the incident and scattered parts, the combined field potential is

$$
\Phi=A \exp (\mathrm{i} \omega t) \sum_{n 0}^{\infty} B_{n}\left[J_{n}(v)+d_{n} H_{n}^{(2)}(v)\right] \cos (n \theta) .
$$

\section{A.4.3. Acoustic force derivation}

Because the solution requires the real component of the potential function, $\phi$ will be rearranged to facilitate the separation into the complex coordinates. With the definition

$d_{n}=a_{n}+\mathrm{i} b_{n}$, where $a_{n}$ and $b_{n}$ are the real and imaginary components of $d_{n}$, respectively, and using Eq. (A.18), the right-hand portion of Eq. (A.20) can be arranged to

$$
\begin{aligned}
& \cos n \theta\left(J_{n}(v)+d_{n} H_{n}^{(2)}(v)\right) \\
& \quad=\cos n \theta\left(J_{n}(v)+\left(a_{n}+\mathrm{i} b_{n}\right)\left(J_{n}(v)-\mathrm{i} Y_{n}(v)\right)\right) \\
& \quad=\cos n \theta\left[\left(1+a_{n}\right) J_{n}(v)+b_{n} Y_{n}(v)+\mathrm{i}\left(b_{n} J_{n}(v)-a_{n} Y_{n}(v)\right)\right] .
\end{aligned}
$$

Defining

$S_{n}=\cos n \theta\left[\left(1+a_{n}\right) J_{n}(v)+b_{n} Y_{n}(v)\right]$,

$T_{n}=\cos n \theta\left[b_{n} J_{n}(v)-a_{n} Y_{n}(v)\right]$,

Eq. (A.20) can be written as

$\Phi=A \exp (\mathrm{i} \omega t) \sum_{n=0}^{\infty} B_{n}\left(S_{n}+\mathrm{i} T_{n}\right)$.

Note that $S_{n}$ and $T_{n}$ are real provided $v>0$ (since the coordinate system is cylindrical, $v=k r$ is always greater than zero and this is satisfied). Also note that $B_{n}$ is real for all $n$ (this may be proved by showing that $B_{n}$ is real for even and odd $n$ separately). With the additional definition of

$$
\begin{aligned}
& W=\sum_{n=0}^{\infty} B_{n} S_{n}, \\
& X=\sum_{n=0}^{\infty} B_{n} T_{n},
\end{aligned}
$$

the velocity potential can be written as

$\Phi=A \exp (\mathrm{i} \omega t)(W+\mathrm{i} X)$,

where $W$ and $X$ are real functions of $v$ and $\theta$. From Eq. (A.12)

$$
P=\operatorname{Re}\left(-\mathrm{i} \omega \rho_{\mathrm{f}} A \exp (\mathrm{i} \omega t)(W+\mathrm{i} X)\right)
$$

or

$P=\omega \rho_{\mathrm{f}} A(W \sin \omega t+X \cos \omega t)$.

The time-averaged square of pressure is

$\left\langle P^{2}\right\rangle=\frac{\left(W^{2}+X^{2}\right) \omega^{2} \rho_{\mathrm{f}}^{2} A^{2}}{2}$.

The expression for the velocity is similarly found to be

$$
\begin{aligned}
\boldsymbol{V}=R e & {\left[A \exp (\mathrm{i} \omega t)\left(\frac{\partial W}{\partial r}+\mathrm{i} \frac{\partial X}{\partial r}\right) \tilde{\boldsymbol{e}}_{r}\right.} \\
& \left.+A \exp (\mathrm{i} \omega t)\left(\frac{1}{r} \frac{\partial W}{\partial \theta}+\mathrm{i} \frac{1}{r} \frac{\partial X}{\partial \theta}\right) \tilde{\boldsymbol{e}}_{\theta}\right] \\
=A[ & {\left[\frac{\partial W}{\partial r} \cos \omega t-\frac{\partial X}{\partial r} \sin \omega t\right) \tilde{\boldsymbol{e}}_{r} } \\
& \left.+\left(\frac{1}{r} \frac{\partial W}{\partial \theta} \cos \omega t-\frac{1}{r} \frac{\partial X}{\partial \theta} \sin \omega t\right) \tilde{\boldsymbol{e}}_{\theta}\right],
\end{aligned}
$$

where $\tilde{\mathbf{e}}_{r}$ and $\tilde{\mathbf{e}}_{\theta}$ are the unit vectors in cylindrical coordinates. From this result, 


$$
\begin{aligned}
\left\langle V^{2}\right\rangle=\frac{A^{2}}{2} & {\left[\left(\frac{\partial W}{\partial r}\right)^{2}+\left(\frac{\partial X}{\partial r}\right)^{2}+\frac{1}{r^{2}}\left(\frac{\partial W}{\partial \theta}\right)^{2}\right.} \\
+ & \left.\frac{1}{r^{2}}\left(\frac{\partial X}{\partial \theta}\right)^{2}\right] \\
= & \frac{k^{2} A^{2}}{2}\left[\left(\frac{\partial W}{\partial v}\right)^{2}+\left(\frac{\partial X}{\partial v}\right)^{2}+\frac{1}{v^{2}}\left(\frac{\partial W}{\partial \theta}\right)^{2}\right. \\
+ & \left.\frac{1}{v^{2}}\left(\frac{\partial X}{\partial \theta}\right)^{2}\right] .
\end{aligned}
$$

With the definitions

$$
\begin{aligned}
& P^{*}=\left(W^{2}+X^{2}\right) \text { and } \\
& V^{*}=\left[\left(\frac{\partial W}{\partial v}\right)^{2}+\left(\frac{\partial X}{\partial v}\right)^{2}+\frac{1}{v^{2}}\left(\frac{\partial W}{\partial \theta}\right)^{2}+\frac{1}{v^{2}}\left(\frac{\partial X}{\partial \theta}\right)^{2}\right],
\end{aligned}
$$

the equation for the acoustic potential (Eq. (A.10)) can be written as

$U=2 \pi R_{\mathrm{p}}^{3} k^{2} A^{2} \rho_{\mathrm{f}}\left(\frac{P^{*}}{6} f_{1}-\frac{V^{*}}{4} f_{2}\right)$.

A dimensionless potential $U^{*}$ is defined as

$$
U^{*}=\frac{U}{4 \pi R_{\mathrm{p}}^{3} E_{\mathrm{ac}}}=\left(\frac{P^{*}}{6} f_{1}-\frac{V^{*}}{4} f_{2}\right) .
$$

For the given coordinate system

$$
\begin{aligned}
& F_{\mathrm{ac}, x^{\prime}}=-F_{\mathrm{ac}, r} \sin \theta-F_{\mathrm{ac}, \theta} \cos \theta \quad \text { and } \\
& F_{\mathrm{ac}, y^{\prime}}=F_{\mathrm{ac}, r} \cos \theta-F_{\mathrm{ac}, \theta} \sin \theta,
\end{aligned}
$$

when Eqs. (A.9), (A.34), and (A.35) are combined, the acoustic forces in the $x$ and $y^{\prime}$ directions are represented by

$$
F_{\mathrm{ac}, x^{\prime}}=4 \pi R_{\mathrm{p}}^{3} E_{\mathrm{ac}} k\left[\frac{\partial U^{*}}{\mathrm{~d} v} \sin \theta+\frac{1}{v} \frac{\partial U^{*}}{\mathrm{~d} \theta} \cos \theta\right],
$$

$$
F_{\mathrm{ac}, y^{\prime}}=-4 \pi R_{\mathrm{p}}^{3} E_{\mathrm{ac}} k\left[\frac{\partial U^{*}}{\mathrm{~d} v} \cos \theta-\frac{1}{v} \frac{\partial U^{*}}{\mathrm{~d} \theta} \sin \theta\right]
$$

\section{A.5. Equations of trajectory motion}

For ease in computation and representation of results, the equations of motion can be cast in terms of the dimensionless variables described in Eq. (3). Once the angle between the approach flow and the incident acoustic field is chosen, it is possible to combine the acoustic force expression (Eqs. (A.36) and (A.37)) with hydrodynamic drag expressions (Eq. (A.2) using Eqs. (A.7) and (A.8)) using the force balance on a single particle (Eq. (A.1)) to determine the trajectory of the particles according to

$\boldsymbol{u}_{\mathrm{p}}=\boldsymbol{u}_{\mathrm{f}}+\frac{\boldsymbol{F}_{\mathrm{ac}}}{6 \pi \mu R_{\mathrm{p}}}$.

\section{References}

[1] S. Gupta, D.L. Feke, AIChE J. 44 (1998) 1005.

[2] S. Gupta, D.L. Feke, Ultrasonics 35 (1997) 131.

[3] D.H. Hill, D.L. Feke, Sep. Sci. Tech. 35 (2000) 1363.

[4] S. Gupta, Ph.D. Thesis, Case Western Reserve University, 1997.

[5] R. Gerber, R.R. Birss, High Gradient Magnetic Separation, John Wiley, New York, 1983.

[6] R. Darby, Chemical Engineering Fluid Mechanics, Marcel Dek ker, New York, 1996.

[7] H. Lamb, Hydrodynamics, sixth ed., Cambridge University Press, London, 1932.

[8] M. Van Dyke, Perturbation Methods in Fluid Mechanics, Parabolic Press, Stanford, CA, 1975.

[9] L.P. Gor'kov, Sov. Phys. Dokl. 6 (1962) 773.

[10] M. Barmatz, P. Collas, J. Acoust. Soc. Am. 77 (1985) 928.

[11] T. Hasegawa, K. Saka, N. Inoue, K. Matsuzawa, J. Acoust. Soc. Am. 83 (1988) 1770.

[12] J. Faran, J. Acoust. Soc. Am. 23 (1951) 405. 